\title{
Vibration safety to reduce the risk of cardiovascular disease in workers
}

\author{
Alexei $\mathrm{Il}^{\prime} \mathrm{ev}^{1}$, Yuri Chertov ${ }^{1}$, Artyom Litvinov², Nataliya Kobzeva ${ }^{3, *}$ \\ ${ }^{1}$ Don State Technical University, 1, pl. Gagarina, 344002, Rostov-on-Don, Russia \\ ${ }^{2}$ Kuban State Technological University, 2, str. Moscow, 350072, Krasnodar, Russia \\ ${ }^{3}$ Rostov State Medical University, 29, Nakhichevan lane, 344022, Rostov-on-Don, Russia
}

\begin{abstract}
Some manufacturing processes are accompanied by significant noise and vibration. Sources of intense noise and vibration are machines and mechanisms with unbalanced rotating masses, as well as technological installations and apparatuses in which the movement of gases and liquids occurs at high speeds and has a pulsating character. The modern development of technology, equipping enterprises with powerful and fastmoving machines and mechanisms leads to the fact that people are constantly exposed to noise of increasing intensity. Increasing noise and vibration in the workplace has a harmful effect on the human body. As a result of prolonged exposure to noise, the normal activity of the cardiovascular and nervous systems, digestive and hematopoietic organs is disturbed, and professional hearing loss develops, the progression of which can lead to complete hearing loss. Among industrial hazards, noise and vibration occupy one of the leading places among production hazards. The harmful effects of increased noise levels on the human body are well khown, therefore, the relevance of this problem is obvious.
\end{abstract}

\section{Introduction}

When working in conditions of vibration, labor productivity decreases, the number of injuries increases. At some workplaces in agricultural production, vibrations exceed normalized values, and in some cases they are close to limit. The vibration levels on the controls do not always comply with the standards. Typically, low-frequency vibrations that negatively affect the body predominate in the vibration spectrum. Some types of vibration adversely affect the nervous and cardiovascular systems, the vestibular apparatus. The most harmful effect on the human body is provided by vibration, the frequency of which coincides with the frequency of natural vibrations of individual organs, the approximate values of which are as follows $(\mathrm{Hz})$ : stomach - $2 \ldots 3$; kidneys - 6 .. 8; heart - 4 ... 6; intestines - $2 \ldots 4$; vestibular apparatus - 0.5 ..L, 3; eyes - $40 \ldots 100$, etc.

The effect on muscular reflexes reaches $20 \mathrm{~Hz}$; the tractor-loaded seat on the tractor has its own vibration frequency of $1.5 \ldots 1.8 \mathrm{~Hz}$, and the rear wheels of the tractor - $4 \mathrm{~Hz}$. The vibration is transmitted to the human body at the moment of contact with the vibrating object: when acting on the limbs, local vibration occurs, and on the whole body - general.

*Corresponding author: 5976765@mail.ru 
Local vibration affects neuromuscular tissues and the musculoskeletal system and leads to spasms of peripheral vessels. With prolonged and intense vibrations, in some cases, a professional pathology develops (local vibration often leads to it): peripheral, cerebral or cerebral-peripheral vibrational disease. In the latter case, there are changes in cardiac activity, general excitement, or, conversely, inhibition, fatigue, the appearance of pain, a feeling of shaking of the internal organs, nausea. In these cases, vibrations also affect the osteoarticular apparatus, muscles, peripheral circulation, vision, hearing $[1,3,5,7,9]$. Local vibrations cause spasms of blood vessels that develop from the terminal phalanges of the fingers, spreading to the entire hand, forearm, and cover the vessels of the heart.

Workers can be exposed to occupational vibration through the use of power or pneumatic hand tools or other machinery, or by driving large transportation, construction or agricultural vehicles. Vibration that is generated through the use of powered hand tools, and is transmitted from the tool to the hand-arm system is referred to as hand-transmitted vibration. However, recent studies have also demonstrated that vibration can be transmitted through platforms workers are standing on, and in these situations, the point of contact is the feet. Workers can also be exposed to whole body vibration (WBV). WBV exposure occurs in occupations where workers are driving trucks, large earth moving vehicles, or where they are using hand tools where the amplitude of the vibration is great enough to be transmitted to other portions of the body, such as in workers using jack-hammers.

Exposure to WBV is of concern within the workforce because it's associated with the development of a number of negative health consequences including back and neck pain, and potentially, cardiovascular disease, the development of various neuropathies, digestive problems, headaches, dizziness, motion sickness and possibly cancer. However, workers exposed to WBV are often also exposed to a number of other risk factors that may contribute to the development of these negative health effects. These risk factors include maintaining a static posture for a long-period of time, torque or twisting of the abdomen to view the area around the vehicle, and heavy lifting that often occurs when a vehicle is being loaded or unloaded. In addition to vibration and the physical exposures associated with a job, there may be other co-exposures to chemicals or certain environmental conditions that contribute to the development of disease or injury in workers. Because most workers are exposed to multiple factors that may induced injury or illness, it's difficult to determine which factors pose the greatest risk for inducing injury or illness. Experimental studies, examining the effects of each of these factor individually on health outcomes can provide additional information that will help determine the contribution of each exposure factor to various health problems.

This review will describe the industrial sectors where vibration exposure is most prevalent and the health effects associated with exposure to HTV and WBV. Experimental methods used to measure and characterize vibration generated in various occupational settings are discussed, and models that have been used to uncover the etiology of vibrationinduced injuries. Although numerous studies have been published on both HTV and HBV, there are new epidemiological studies showing an increased risk of specific cancers with exposure to WBV. Therefore additional etiological studies need to be performed. New avenues for research are discussed below.

\section{Methods}

The human body is considered as a combination of masses with elastic elements. In one case, it is the whole body with the lower part of the spine and the pelvis, in the other - the upper part of the body in combination with the upper part of the spine, inclined forward. For a person standing on a vibrating surface, there are 2 resonant peaks at frequencies of 5 $\ldots 12$ and $17 \ldots 25 \mathrm{~Hz}$, for a person sitting at frequencies of $4 \ldots 6 \mathrm{~Hz}$. For the head, the 
resonant frequencies are in the range of $20 \ldots 30 \mathrm{~Hz}$. In this frequency range, the amplitude of head vibrations can exceed the amplitude of shoulder vibrations by 3 times. Fluctuations in the internal organs, chest and abdominal cavity show resonance at frequencies of $3.0 \ldots$ $3.5 \mathrm{~Hz}$ (Fig. 1).
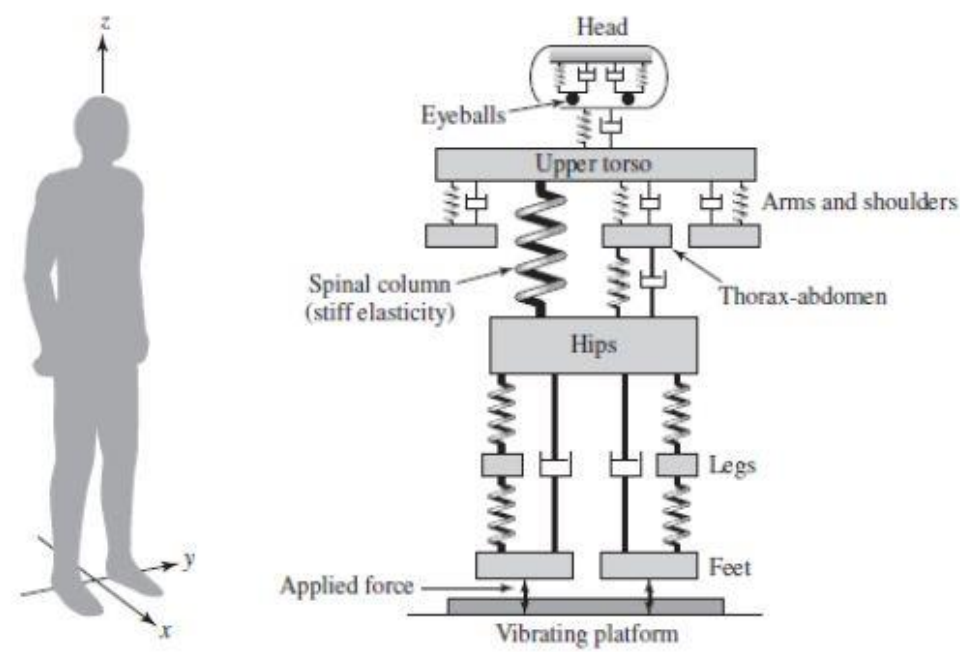

Fig. 1. Human vibration pattern.

The maximum vibration amplitude of the abdominal wall is observed at frequencies of 7 ... $8 \mathrm{~Hz}$. With an increase in the frequency of oscillations, their amplitude decreases during transmission through the human body. In the standing and sitting position, these attenuations on the pelvic bones are $9 \mathrm{~dB}$ per octave of frequency change, on the chest and head $-12 \mathrm{~dB}$, on the shoulder $-12 \ldots 14 \mathrm{~dB}$. These data do not apply to resonant frequencies, under the influence of which there is not attenuation, but an increase in the vibrational velocity.

Under industrial conditions, hand-held machines whose vibration has maximum energy levels (maximum vibration velocity) in the low-frequency bands (up to $36 \mathrm{~Hz}$ ) cause vibration pathology with predominant damage to the neuromuscular tissue and musculoskeletal system. When working with manual machines, the vibration of which has a maximum energy level in the high-frequency region of the spectrum (above $125 \mathrm{~Hz}$ ), mainly vascular disorders occur. When exposed to low-frequency vibration, the disease occurs after $8 \ldots 10$ years, and when exposed to high-frequency vibration - after 5 years and earlier. The general vibration of different parameters causes a different degree of severity of changes in the nervous and system (central and autonomic), cardiovascular system and vestibular apparatus.

Depending on the parameters (frequency, amplitude), vibration can both positively and negatively affect individual tissues and the body as a whole. Vibration is used in the treatment of certain diseases, but most often vibration (production) is considered a harmful factor. Therefore, it is important to know the boundary characteristics that separate the positive and negative effects of vibration on humans. For the first time, the French scientist Abbot Saint Pierre drew attention to the useful value of vibration, who in 1734 constructed a vibrating chair for couch potatoes, which increases muscle tone and improves blood circulation. At the beginning of the XX century. in Russia, professor of the Military Medical Academy A.E. Scherbak proved that moderate vibration improves tissue nutrition and accelerates wound healing[11]. 
Industrial vibration, characterized by a significant amplitude and duration of action, causes workers irritability, insomnia, headache, aching pains in the hands of people dealing with a vibrating tool. With prolonged exposure to vibration, bone tissue is rebuilt: on radiographs, stripes similar to fracture traces can be seen - areas of greatest tension where bone tissue softens. The permeability of small blood vessels increases, nervous regulation is disturbed, and the sensitivity of the skin changes. When working with a mechanized hand tool, acroasphyxia (a symptom of dead fingers) can occur - loss of sensitivity, whitening of fingers, hands. Under the influence of general vibration, changes from the central nervous system are more pronounced: dizziness, tinnitus, memory impairment, impaired coordination of movements, vestibular disorders, weight loss appear (Fig.2).

Main vibration parameters: frequency and amplitude of vibrations. A point oscillating with a certain frequency and amplitude moves with continuously changing speed and acceleration: they are maximum at the moment of its passage through the initial rest position and decrease to zero in extreme positions. Therefore, the oscillatory motion is also characterized by speed and acceleration, which are derivatives of amplitude and frequency. Moreover, the human senses perceive not the instantaneous value of the vibration parameters, but the current one $[2,4,6,8,10]$.

i_c $(1)=0.000$ Eigenfrequency $=8.369$ Surface: Total displacement $(\mathrm{m})$

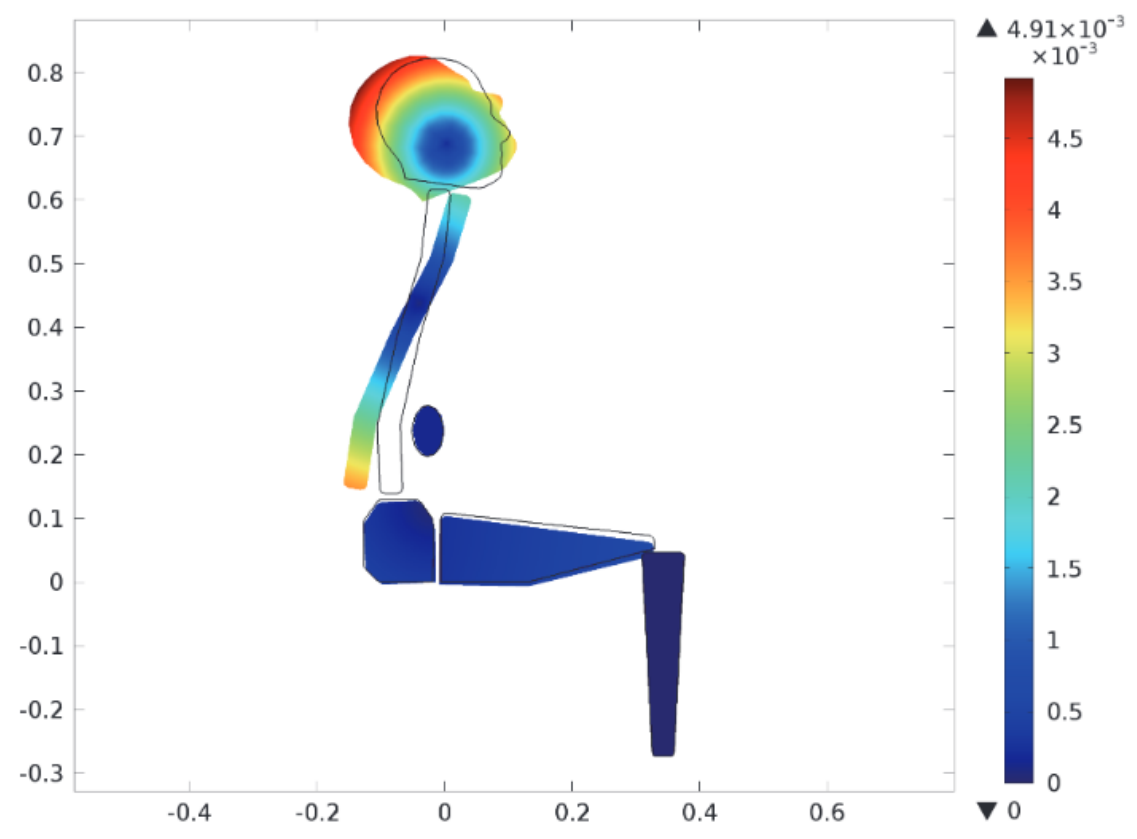

Fig. 2. Biomechanical model of vibration perception.

Vibration is often measured with instruments whose scales are not graded in absolute values of speed and acceleration, but in relative decibels. Therefore, vibrational level and vibrational acceleration level also serve as vibration characteristics. Considering a person as a complex dynamic structure with time-varying parameters, it is possible to distinguish frequencies that cause a sharp increase in the amplitudes of oscillations of the whole body as a whole and of its individual organs. With vibration below $2 \mathrm{~Hz}$, acting on a person along the spine, the body moves as a whole. Resonant frequencies depend little on the individual characteristics of people, since the main subsystem that responds to vibrations is 
the abdominal organs that vibrate in one phase. Resonance of internal organs occurs at a frequency of $3 \ldots 3.5 \mathrm{~Hz}$, and at $4 \ldots 8 \mathrm{~Hz}$ they are displaced.

\section{Results}

Vibration isolation is to reduce the transmission of vibrations from the source to the protected object using devices placed between them. For vibration isolation, vibration isolating supports such as elastic gaskets, springs, or a combination thereof are most often used. The effectiveness of vibration isolators is evaluated by the gearbox transmission coefficient equal to the ratio of the amplitude of vibration displacement, vibration velocity, vibration acceleration of the protected object, or the force acting on it to the corresponding parameter of the vibration source. Vibration isolation only reduces vibration when the gearbox is $<1$. The smaller the gearbox, the more effective the vibration isolation.

The means of protection against vibrations are divided into: collective and individual. The main measures to protect against vibration can conditionally be reduced to such groups: technical, organizational, and treatment and prophylactic.

Technical measures include: eliminating vibrations in the source and on the path of their propagation. Elimination or reduction of vibration in the source is decided, starting from the stage of design and manufacture of machines. Solutions that provide vibration-free working conditions are laid in their design: replacing shock processes with shock-free, using plastic parts, belt drives instead of chain, gears with globoidal and chevron gearing instead of spur gears, choosing optimal operating modes, carefully balancing rotating parts, improving their accuracy class manufacturing and surface finish cleanliness and more.

During operation of the equipment, reduced vibrations are achieved by modern tightening of fasteners, elimination of backlash, gaps, high-quality lubrication of rubbing surfaces, proper adjustment of working bodies.

In constructions through which the propagation of vibrations occurs, gaps are made, filled with vibration and soundproof materials; replacement of vibrating equipment or process with vibration-free.

To reduce vibrations along the propagation path, apply: vibration isolation, vibration damping, vibration damping.

Vibration isolation:

In engineering practice, one of the effective measures to reduce vibrations along the path of its propagation from a vibration source is vibration isolation. Vibration isolation is passive and active.

Vibration isolation is called active if an additional energy source is used to reduce it.

Passive vibration isolation is used if it is necessary to protect the workplace from vibrations of vibrating machines or to protect other machines from vibrations of unbalanced parts (SSBT GOST 12.4.046-78 "Methods and means of vibration protection. Classification.").

Vibration isolation weakens the transmission of vibrations from the source to the base, floor, workplace, etc. due to the elimination of hard bonds between them and the installation of elastic elements (vibration isolators).

If technical means cannot achieve hygiene standards at the workplace, then personal protective equipment must be used: vibration-proof gloves and vibration-proof shoes, kneecaps, rugs, bibs, and special suits. The vibration-protective properties of the applied elastic materials are normalized in octave bands of $8 \ldots 2000 \mathrm{~Hz}$ and should be within $1 \ldots 5$ $\mathrm{dB}$ with an insert thickness of $5 \mathrm{~mm}$ and $1 \ldots 6 \mathrm{~dB}$ with an insert thickness of $10 \mathrm{~mm}$. The pressure force when evaluating the vibration-protective properties of mittens varies from 50 to $200 \mathrm{~N}$. The vibration-proof mittens must be hygienic, not impede the performance of 
technological operations, and not cause irritation of the skin (GOST 12.4 002-74 "Personal protective equipment for hands on vibration. General technical requirements") .

Vibro-insulating shoes are made of leather (or artificial substitutes) and equipped with insoles of elastoplastic materials to protect against vibration at frequencies above $11 \mathrm{~Hz}$. The effectiveness of vibration-proof shoes is standardized at frequencies of $16 ; 31.5 ; 63 \mathrm{~Hz}$ and should be $7 \ldots 10 \mathrm{~dB}$. The requirement for the manufacture of vibration-proof shoes and methods for determining protective effectiveness are given in GOST 12.4.024-76 * "Special vibration-proof shoes. General technical requirements. "

Organizational and preventive measures to reduce the harmful effects of vibration include the rational mode of work and rest and the use of therapeutic and preventive measures. When working with a tool with fluctuations up to 1200 per minute, workers need a 10-minute break after each hour of work; when working with a tool having 4000 or more vibrations per minute, a half-hour break is required after each hour of operation.

Avoid exposure to vibration for more than $65 \%$ of the working time. According to sanitary standards, it is forbidden to work with a pneumatic tool at temperatures below 160 ${ }^{\circ} \mathrm{C}$, humidity $40-60 \%$ and air speed over $0.3 \mathrm{~m} / \mathrm{s}$.

When working with a vibrating tool to prevent disease, the mass of the instrument held in the hands should not exceed $10 \mathrm{~kg}$, and the pressure force working on the vibrating equipment should not exceed $200 \mathrm{~N}$

Preventive measures to protect against vibration are to reduce them in the source of education and on the path of distribution, as well as in the use of personal protective equipment, sanitary and organizational measures.

Vibration reduction in the source of occurrence is achieved by a change in the technological process with the manufacture of parts from nylon, rubber, textolite, timely preventive measures and lubrication operations; centering and balancing parts; reduction of gaps in joints. The transmission of vibrations to the base of the unit or the building structure is weakened by shielding, which is both a means of combating noise.

As vibration absorbing coatings, mastics No. 579, 580, type BD-17, and simplest structures (roofing layers glued with bitumen or synthetic glue) are usually used. If collective protection methods do not give a result or are irrationally applied, then personal protective equipment is used. As protection against vibration when working with a mechanized tool, anti-vibration gloves and special shoes are used. Anti-vibration ankle boots have a multi-layer rubber sole.

The duration of work with a vibrating tool should not exceed $2 / 3$ of the work shift. The operations are distributed among the workers so that the duration of continuous vibration, including micro-pauses, does not exceed $15 \ldots 20$ minutes. It is recommended to take breaks for 20 minutes $1 \ldots 2$ hours after the start of the shift and for 30 minutes 2 hours after lunch.

During breaks, a special set of gymnastic exercises and hydroprocedures should be performed - baths at a water temperature of $38^{\circ} \mathrm{C}$, as well as self-massage of limbs.

If the vibration of the machine exceeds the permissible value, then the contact time of the person working with this machine is limited.

To increase the protective properties of the body, working capacity and labor activity, special complexes of industrial gymnastics, vitamin prophylaxis (twice a year a complex of vitamins $\mathrm{C}$, B, nicotinic acid), special nutrition should be used.

\section{Discussion}

The influence of noise and vibration on a person and his body in recent decades has become one of the most urgent problems in all countries of the world. Noise affects a person in 
production (meaning industrial enterprises and some noise objects), the street and in the house.

From an unsatisfactory state of affairs with life safety, the country annually suffers large human, financial, economic, material and moral losses. Ensuring the safety of production and labor protection of workers is one of the main problems of the national security of the country. At present, in our country, many enterprises do not observe safety precautions, and working conditions cannot be called favorable.

Under the influence of intense noise and vibration, fatigue and irritability, poor sleep, headache, weakening of memory, attention and visual acuity occur, which leads to a decrease in labor productivity (on average by 10-15\%) and is often the cause of injuries. Vibration and noise affect the cardiovascular, endocrine and nervous systems, disrupt the coordination of movements. Adaptation to noise is not possible.

\section{Acknowledgements}

The study had no sponsorship.

\section{References}

1. A.T. Rybak, V.P. Zharov, A.V. Serdyukov, Russian Engineering Research 29(2), 194197 (2009)

2. A. Rybak, O. Lyakhnitskaya, MATEC Web of Conferences 132, 01001 (2017)

3. A.T. Rybak, A.R. Temirkanov, O.V. Lyakhnitskaya, Russian Engineering Research 38(9), 702-704 (2018)

4. A.T. Rybak, A.R. Temirkanov, O.V. Lyakhnitskaya, Russian Engineering Research 38(3), 212-217 (2018)

5. K. Kobzev, S. Shamshura, A. Chukarin, V. Bogdanovich, V. Kasyanov, MATEC Web of Conferences 226, 01022 (2018)

6. K. Kobzev, S. Shamshura, A. Chukarin, A. Buryanovand, V. Kasyanov, MATEC Web of Conferences 226, 01023 (2018)

7. K. Kobzev, A. Chukarin, IOP Conference Series: Earth and Environmental Science 403, 012145 (2019)

8. K.O. Kobzev, E.S. Bozhko, A.V. Mozgovoi, M.D. Molev, N.I. Stuzhenko, IOP Conference Series: Materials Science and Engineering 680, 012014 (2019)

9. K.O. Kobzev, E.S. Bozhko, A.V. Mozgovoi, E.I. Kostromina, L.G. Babenko, IOP Conference Series: Materials Science and Engineering 680, 012013 (2019)

10. N. Kobzeva, V. Terentev, I. Zolotuhina, IOP Conference Series: Materials Science and Engineering 680, 012046 (2019)

11. N. Kobzeva, V. Terentev, I. Zolotuhina, IOP Conference Series: Materials Science and Engineering 680, 012011 (2019) 